\title{
Constructive Semi-Supervised Classification Algorithm and Its Implement in Data Mining
}

\author{
Arvind Singh Chandel ${ }^{1}$, Aruna Tiwari ${ }^{1}$, and Narendra S. Chaudhari ${ }^{2}$ \\ ${ }^{1}$ Department of Computer Engg. \\ Shri GS Inst of Tech.\& Sci. \\ SGSITS, 23, Park Road, \\ Indore (M.P.) India 452001 \\ atiwari@sgsits.ac.in, asc_chandel@rediffmail.com \\ ${ }^{2}$ Department of Computer Science and Engineering (CSE) \\ IIT, Indore \\ M-Block, IET-DAVV Campus, \\ Khandwa Road, Indore-452017(M.P.) \\ NSchaudhari@ittbombay.org, NSC183@gmail.com
}

\begin{abstract}
In this paper, we propose a novel fast training algorithm called Constructive Semi-Supervised Classification Algorithm (CS-SCA) for neural network construction based on the concept of geometrical expansion. Parameters are updated according to the geometrical location of the training samples in the input space, and each sample in the training set is learned only once. It's a semisupervised based approach, the training samples are semi-labeled i.e. for some samples, labels are known and for some samples, data labels are not known. The method starts with clustering, which is done by using the concept of geometrical expansion. In clustering process various clusters are formed. The clusters are visualizes in terms of hyperspheres. Once clustering process over labeling of hyperspheres is done, in which class is assigned to each hypersphere for classifying the multi-dimensional data. This constructive learning avoids blind selection of neural network structure. The method proposes here is exhaustively tested with different benchmark datasets and it is found that, on increasing value of training parameters number of hidden neurons and training time both are getting decrease. Through our experimental work we conclude that CS-SCA result in simple neural network structure by less training time.
\end{abstract}

Keywords: Semisupervised classification, Geometrical Expansion, Binary Neural Network, Hyperspheres.

\section{Introduction}

Constructive learning begins with a minimal or empty structure, and dramatically increases the network by adding hidden neurons until a satisfactory solution is found. Numbers of Constructive learning algorithms are available to overcome the problem of traditional algorithms for classification. It includes Fast Covering Learning Algorithm (FCLA) for Supervised Learning [2], Constructive Unsupervised Learning 
Algorithm (CULA) [1], Constructive set Covering learning algorithm (CSCLA) by Ma and Yang [4], Boolean-like training algorithm (BLTA) by Gray and Michel [5], expand and truncate learning algorithm (ETL) by Kim and Park [3]. BLTA is a dynamic technique and derives its original principle from Boolean algebra with extension. ETL finds a set of required separating hyperplanes and automatically determines a required number of neurons in the hidden layer based on geometrical analysis of the training set. CSCLA was proposed based on the idea of weighted Hamming distance hypersphere. In general, ETL, IETL, CSCLA, have no generalization capability. BLTA has generalization capability, but needs more hidden neurons.

Moreover to it, FCLA is supervised learning algorithm, thus labeled samples are used for learning but labeled data sample are expensive to obtain as they require the effort of experienced human annotators. On the other hand unlabeled data samples are easy to obtain but there are very few way to process them. Thus approach of semisupervised was used that uses large amount of unlabeled data sample with small amount of labeled data sample to build the classifier.

In this paper, we propose a novel fast training algorithm called Constructive semisupervised classification Approach (CS-SCA) for neural network construction. The proposed method is implemented using two processes, first is clustering and second is labeling. We illustrate the advantages of CS-SCA by using it in classification problems. There are various features of CS-SCA like it is a semi-supervised constructive approach. Sample reordering is allowed in proposed classifier and because of reordering, learning is fast in this approach. As we know that CS-SCA is a semi-supervised approach that's why it requires less human effort. This CS-SCA approach is tested with number of benchmark datasets and compared with SVM [6] based classifier.

The paper is organized as follows. Section 2 gives an overview of CS-SCA. Section 3 explains the method for CS-SCA in detail give algorithmic formulation of our methodology. In section 4, we give experimental results to demonstrate the usefulness of our approach; it also contains detail of data preparation. These experimental results include two well-known datasets [7], namely, Riply dataset and Wisconsin Breast cancer dataset. Finally, in section 5, we give concluding remarks.

\section{Overview of CS-SCA}

\subsection{Basic Concept}

Boolean functions have the geometrical property which makes it possible to transform non-linear representation to linear representation for each hidden neuron. We consider a Boolean function with $\mathrm{n}$ input and one output,

$$
\begin{gathered}
\qquad y=f\left(x_{1}, x_{2}, \ldots, x_{n}\right), \\
\text { Where } y \in(0,1) \text { and } x_{i} \in(0,1), i=(1 \ldots . n) .
\end{gathered}
$$

These $2^{n}$ binary patterns $(0,1)^{n}$ can be considered as a $n$-dimensional unit hypercube.This ex-hypersphere is defined as the reference hypersphere (RHS) [5] as follows:

$$
\left(x_{1}-1 / 2\right)^{2}+\left(x_{2}-1 / 2\right)^{2}+\ldots+\left(x_{n}-1 / 2\right)^{2}=n / 4 \text {. }
$$




\subsection{Network Construction}

CS-SCA constructs a three-layered feed forward neural network with an input layer, a hidden layer and an output layer, as shown in Fig-1. We illustrate the advantages of CS-SCA by its implement in classification problems.

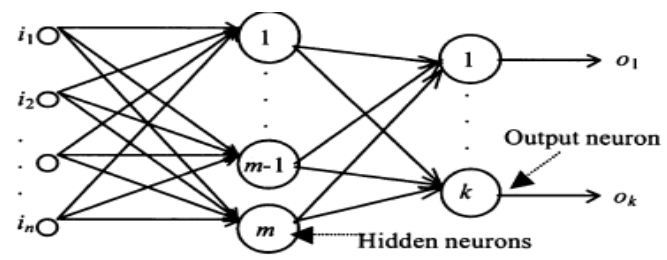

Fig. 1. Neural Network Structure by CS-SCA

\section{Proposed Method: CS-SCA}

CS-SCA begins with an empty hidden layer. To learn a sample, CS-SCA either adds a new hidden neuron to represent it or updates the parameters of some hidden neuron by expanding its corresponding hypersphere. This is done by clustering process and once clustering gets over by using the concept of majority voting labeling of hypersphere is done.

\subsection{Clustering Process}

CS-SCA constructs a three-layered feed forward neural network, of which first layer represent to the input data sample that will be in the binary coded format. Then input data samples will be grouped into various clusters. The middle layer of network architecture represents the hyperspheres(hidden neuron).A hidden neuron in Fig. 1 represents a corresponding hyper sphere with center $\mathrm{c}$ and radius $r_{1}$. While constructing a hidden neuron, suppose that $\left\{x^{1}, x^{2}, \ldots, x^{v}\right\}$ are $\mathrm{v}$ (true) sample included in one hyper sphere (hidden neuron). In terms of these samples, the center is defined as the gravity center $c=\left(c_{1}, c_{2}, \ldots, c_{n}\right)$;

$$
c_{i}=\sum_{k=1}^{v} \frac{x_{i}^{k}}{v}
$$

The radius $r_{1}$ is defined as the minimal Euclidean distance such that all the v vertices are exactly in or on the surface of the corresponding hyper sphere.

$$
r_{1}=\min _{j=1}^{v}\left\|x^{j}-c\right\|=\min _{j=1}^{v}\left(\sum_{i=1}^{n}\left(x_{i}^{k}-c_{i}\right)^{2}\right)^{1 / 2}
$$

Where $\mathrm{n}$ is the dimension of the input and $\mathrm{I}^{*} \|$ is the euclidean distance.

Given $c$ and $r_{1}$ we can separate these $\mathrm{v}$ true sample from the remaining samples. In another words, this corresponding hypersphere represents these $\mathrm{v}$ true samples. 
Two secondary central radii $r_{2}$ and $r_{3}$ are introduced to find compact cluster. Samples should be a compact cluster where $r_{1}<r_{2}<r_{3}$.

CS-SCA begins with an empty hidden layer. To construct the neural network, we examine whether a coming "true" sample can be covered by one of the existing hidden neurons. When the first sample $x^{1}$ comes, the hidden layer is empty and no hidden neuron covers this sample. A new hidden neuron, the first hidden neuron, is created to represent it. This new created hidden neuron represents a hyper sphere centered at $x^{1}$. Samples, which have been represented, are removed after parameter updating. The training process goes on. A coming sample $x^{k}$ causes one of the following actions.

1. Update the parameters of some hidden neuron, and remove $\mathrm{c}$;

2. Create a new hidden neuron to represent it, and remove $x^{k}$;

3. Back up $x^{k}$ to be learned in the next training circle.

Given a hidden neuron $\mathrm{j}$ with the center $c^{j}$ and three $\operatorname{radii} r_{1}^{j}, r_{2}^{j}$ and $r_{3}^{j}$, we firstly compute the function for the hidden neuron $\mathrm{j}$ defined as:

$$
f\left(w^{j}, x^{k}\right)=\sum_{i=1}^{n} w_{i}^{j} x_{i}^{k}
$$

Where $w^{j}$ is $\left(w_{1}^{j}, w_{2}^{j}, \ldots, w_{n}^{j}\right)$, the weight vector and $x^{k}$ is the $k^{t h}$ vertex. The training process is continued as follows:

I. If $f\left(w^{j}, x^{k}\right) \geq t_{1}^{j}$, already covered, so nothing needs to be done.

II. If $t_{1}^{j}>f\left(w^{j}, x^{k}\right) \geq t_{2}^{j}$ the sample $\mathrm{x}$ is within the "claim region"; so to include it an immediate expansion is needed.

III. If $f\left(w^{j}, x^{k}\right) \geq t_{3}^{j}$ the sample is confusing sample so back up $x^{k}$ to be dealt with in the next training circle.

IV. If for all j's, $f\left(w^{j}, x^{k}\right)<t_{3}^{j}$ then create a new hidden neuron and remove the sample $x^{k}$ from the training set.

Thus the number of neurons generated is equal to the number of clusters. After this, the labeled samples are useful for labeling the clusters. The details are given next.

\subsection{Labeling Process}

In labeling process labels are assigned to hyperspheres formed after the clustering process by using the mechanism of Majority voting concept. Thus these labeled hypersphere can be represented as output neuron in the output layer of network architecture. After clustering when hyperspheres are identified, we assign labels to hyperspheres.

1. Repeat the step 2 and step 3 for each of the hyper sphere.

2. Perform majority voting by count number of samples belongs to one particular class. 
3. Majority of samples of particular class in an individual hypersphere would decide the class of that hypersphere.

4. If a particular hypersphere is not covering any labeled data in that case merge this hypersphere with other which is closure to it.

\section{Experimental Work}

We used a Personal Computer (PC) with Pentium processor with $2.99 \mathrm{GHz}$ speed and $1 \mathrm{~GB}$ of RAM having windows XP operating system for testing. We used Matlab 7.0.1 for implementation.

Table 1.

\begin{tabular}{cccc}
\hline Dataset & $\begin{array}{c}\text { Dimensions, Number of } \\
\text { classes }\end{array}$ & Training & Testing \\
\hline Fisher's Iris & 4,3 & 123 & 27 \\
Breast Cancer & 9,2 & 683 & 68 \\
Balance Scale & 4,3 & 492 & 76 \\
Riply & 2,2 & 720 & 80
\end{tabular}

Each training samples $x^{k}=\left(x_{1}^{k}, x_{2}^{k}, \ldots, x_{n}^{k}\right)$ are normalized as follows:

$$
x_{i}^{k}=x_{i}^{k}-\min \left(x_{i}\right) / \max \left(x_{i}\right)-\min \left(x_{i}\right)
$$

After this transformation, each data sample is transformed in the range 0 and 1 . CSSCA requires binary form of input data therefore after normalization re-quantizes the data into eight levels as follows.

1. Apply each sample as an input in quantized function given in step 3 .

2. Quantized value can be obtained by: $y=$ uencode $(u, n, v)$

3. Repeat step 3, till the whole sample binary coded

After data preparation, for experimentation $80 \%$ of the original data taken as training data and rest $20 \%$ considered as testing samples. The datasets used for experimentation are given in table 1 . Results are evaluated in terms of classification accuracy, training time, confusing samples and number of hyperspheres required. For different value of training parameter results for each dataset are getting change. After calculating the performance of CS-SCA, same datasets are applied in SVM based classifier [6], to compare the performance of both the classifiers, in terms of Classification accuracy, Training time. In SVM based classifier, training parameter $\alpha$ used in clustering process. Number of support vector in SVM based classifier depends on the value of training parameter $\alpha$. Comparison results of both the classifier are displayed in Table3. 
Table 2. For 10-fold cross validation results

\begin{tabular}{cc}
\hline Dataset & Average Accuracy \\
\hline $\begin{array}{c}\text { Wisconsin } \\
\text { Beast Cancer } \\
\text { Riply }\end{array}$ & $85.1 \%$ \\
& $80.1 \%$ \\
\hline
\end{tabular}

Table 3. Comparison with SVM

\begin{tabular}{ccccc}
\hline Dataset & $\begin{array}{c}\text { Accuracy } \\
\text { by CS- } \\
\text { SCA }\end{array}$ & $\begin{array}{c}\text { Accuracy } \\
\text { in SVM }\end{array}$ & $\begin{array}{c}\text { Training } \\
\text { Time in } \\
\text { CS-SCA }\end{array}$ & $\begin{array}{c}\text { Training } \\
\text { Time in } \\
\text { SVM }\end{array}$ \\
\hline Fisher's Iris & $92.59 \%$ & $77 \%$ & $0.96 \mathrm{sec}$. & $5.89 \mathrm{sec}$. \\
$\begin{array}{c}\text { Balance } \\
\text { Scale }\end{array}$ & $80.26 \%$ & $77 \%$ & $2.18 \mathrm{sec}$. & $65.9 \mathrm{sec}$. \\
$\begin{array}{c}\text { Wisconsin } \\
\text { breast cancer } \\
\text { Riply }\end{array}$ & $85 \%$ & $70 \%$ & $4.29 \mathrm{sec}$. & $1086 \mathrm{sec}$. \\
\hline
\end{tabular}

We give results for 10-fold cross validation on Wisconsin Breast Cancer and Riply dataset in table 2 shown above. For 10-fold cross validation $90 \%$ of the data taken as training and rest $10 \%$ taken as testing data. From the results shown in above table 3 , it's clear that for each dataset CS-SCA is giving better accuracy and requires less training time compare to SVM based classifier.

\section{Concluding Remarks}

A binary neural network based Semi-supervised classifier is constructed using the concept of geometrical expansion, which classify semi-labeled data. The classification is performed using two processes, first is clustering and second is labeling. Various benchmark datasets used to demonstrate the performance of CS-SCA in terms of accuracy and number of hypersphere etc. After that same datasets is applied in SVM based classifier to compare its performance with developed classifier. It's found that CS-SCA gives better performance in terms of accuracy, training time etc.

\section{References}

1. Wang, D., Chaudhari, N.S.: A Constructive Unsupervised Learning Algorithm for Clustering Binary Patterns. In: Proceedings of International Joint Conference on Neural Networks (IJCNN 2004), Budapest, July 2004, vol. 2, pp. 1381-1386 (2004)

2. Wang, D., Chaudhari, N.S.: A Novel Training Algorithm for Boolean Neural Networks Based on Multi-Level Geometrical Expansion. Neurocomputing 57C, 455-461 (2004)

3. Kim, J.H., Park, S.K.: The geometrical learning of binary neural neworks. IEEE Transaction. Neural Networks 6, 237-247 (1995)

4. Joo Er, M., Wu, S., Yang, G.: Dynamic Fuzzy Neural Networks. McGraw-Hill, New York (2003)

5. Kwok, T.Y., Yeung, D.Y.: Constructive algorithms for structure learning in feedforward neural networks for regression problems. IEEE Trans. Neural Networks 8, 630-645 (1997)

6. Chaudhari, N.S., Tiwari, A., Thomus, J.: Performance Evaluation of SVM Based Semisupervised Classification Algorithm. In: International Conference on Control, Automation, Robotics and Vision, Hanoi, Vietman, December 17-20 (2008)

7. http://www.ics.uci.edu/kmlearn/MLRepository.html 